PROCEEDINGS OF THE

AMERICAN MATHEMATICAL SOCIETY

Volume 124, Number 2, February 1996

\title{
COMPLETELY POSITIVE MODULE MAPS AND COMPLETELY POSITIVE EXTREME MAPS
}

\author{
SZE-KAI TSUI
}

(Communicated by Palle E. T. Jorgensen)

\begin{abstract}
Let $A, B$ be unital $C^{*}$-algebras and $P_{\infty}(A, B)$ be the set of all completely positive linear maps of $A$ into $B$. In this article we characterize the extreme elements in $P_{\infty}(A, B, p), p=\Phi(1)$ for all $\Phi \in P_{\infty}(A, B, p)$, and pure elements in $P_{\infty}(A, B)$ in terms of a self-dual Hilbert module structure induced by each $\Phi$ in $P_{\infty}(A, B)$. Let $P_{\infty}(B(H))_{R}$ be the subset of $P_{\infty}(B(H), B(H))$ consisting of $R$-module maps for a von Neumann algebra $R \subseteq B(\mathbb{H})$. We characterize normal elements in $P_{\infty}(B(H))_{R}$ to be extreme. Results here generalize various earlier results by Choi, Paschke and Lin.
\end{abstract}

\section{INTRODUCTION}

Let $A, B$ be $C^{*}$-algebras and $P_{\infty}(A, B)$ be the set of completely positive linear maps of $A$ into $B$. In case $A=B$ we write $P_{\infty}(A)$ for $P_{\infty}(A, A)$. In case $A$ is unitial, we let $P_{\infty}(A, B, p)$ be a subset of $P_{\infty}(A, B)$ consisting of elements $\Phi$ in $P_{\infty}(A, B)$ with $\Phi(I)=p$. Recently, there has been renewed interest in studying elements $\Phi$ in $P_{\infty}(A, B)$ to be pure [9], [16] to satisfy a null-kernel condition [15], [16] and elements in $P_{\infty}(A, B, p)$ to be extreme [3], [4], [8], [10], [16]. Earlier works on these subjects can be found in Stфrmer [13], Arveson [1], Choi [2], Paschke [11], Kraus [7], Choi and Lam [3], Takesaki and Tomiyama [14]. Here we don't intend to include all references on the subject but only a few more relevant representatives.

Let $R$ be a von Neumann algebra acting on a Hilbert space $\mathbb{H}$. We denote by $L_{\infty}(B(\mathbb{H}))_{R}$ (respectively $P_{\infty}\left(B(\mathbb{H})_{R}\right)$ the set of all completely bounded (respectively positive) $R$-module maps on $B(\mathbb{H})$. In 1980 , Haagerup described all normal elements in $L_{\infty}(B(\mathbb{H}))_{R}$. Since $P_{\infty}(B(\mathbb{H}), p)_{R}$ is a subset of $L_{\infty}(B(\mathbb{H}))_{R}$, based on Haagerup's result, we are able to describe all normal extreme elements in $P_{\infty}(B(\mathbb{H}), p)_{R}$ in section 2 .

Let $M_{n}$ be the $n \times n$ matrix algebra over complex numbers. It was shown by Choi in [2] that elements $\Phi$ in $P_{\infty}\left(M_{n}, M_{m}\right)$ are of the form $\Phi(x)=\sum_{i=1}^{k} a_{i}^{*} x a_{i}$, for all $x \in M_{n}$, where $a_{i}$ is an $n \times m$ matrix for $i=1, \ldots, k$, and $\Phi$ is extreme in $P_{\infty}\left(M_{n}, M_{m}, p\right)$ if and only if $\left\{a_{i}^{*} a_{j}\right\}_{i, j}$ is linearly independent. Haagerup showed that normal elements $\Phi$ in $L_{\infty}(B(\mathbb{H}))_{R}$ are of the form $\Phi(x)=\sum_{i=1}^{\infty} a_{i} x b_{i}$ convergent in the strong-operator topology with $a_{i}, b_{i}$ in $R^{\prime}$, the commutant of $R$. In

Received by the editors July 15, 1994.

1991 Mathematics Subject Classification. Primary 46L05, 46L40.

Key words and phrases. Pure completely positive linear maps, extreme completely linear maps, module maps, strongly independent, Hilbert module representations. 
section 2 we show that such a completely positive $\Phi$ is extreme in $P_{\infty}(\mathbb{B}(\mathbb{H}), p)_{R}$ if and only if $\left\{a_{i}^{*} a_{j}\right\}_{i, j}$ is strongly independent (see Theorem 2.3). This result can be viewed as the analytic generalization of the result by Choi in the finite case. Our result here also generalizes results of Delaroche in [4].

In section 3 we establish characterizations of pure elements in $P_{\infty}(A, B)$ and extreme elements in $P_{\infty}(A, B, p)$. The characterizations we give here are generalizations of those in Paschke's paper [11] and Lin's paper [9]. In [11] Paschke characterized pure elements and extreme elements in $P_{\infty}(A, B)$ and $P_{\infty}(A, B, p)$ respectively for von Neumann algebras $B$, because the Hilbert $B$-module induced by an element in $P_{\infty}(A, B)$ can be made self-dual for von Neumann algebras $B$. It is known that such a Hilbert $B$-module is not necessarily self-dual, if $B$ is not a von Neumann algebra. In section 3 , we find a Hilbert $\bar{B}$-module, induced by an element in $P_{\infty}(A, B)$ for a $\sigma$-closure $\bar{B}$ of $B$ such that this Hilbert $\bar{B}$-module is self-dual. Then we can characterize pure and extreme elements in $P_{\infty}(A, B)$ and $P_{\infty}(A, B, p)$ respectively through such an induced self-dual Hilbert module and the module representation. The concepts of (extreme) completely positive linear maps have been studied and used by quantum physicists for a long time as indicated in [6]. Recently, the topic of module maps has been hotly pursued. It is not my intent to give a list of general references on the topic except for a couple which are actually used in our deliberation.

Finally, I would like to thank Huaxin Lin for sending me the preprint [8] and Erling Størmer and Larry Brown for the conversation on this topic during the final stage of this work.

\section{Normal extreme COMPletely positive maps on $B(\mathbb{H})$}

2.1 Notation. We denote the set of square summable sequences of vectors in $\mathbb{H}$ by $\ell_{2}(\mathbb{H})$, i.e., $\ell_{2}(\mathbb{H})=\left\{\left(x_{i}\right)_{i} \mid x_{i} \in \mathbb{H} \& \sum\left\|x_{i}\right\|^{2}<\infty\right\}$, and note that $\ell_{2}(\mathbb{H})=\ell_{2} \otimes \mathbb{H}$ as Hilbert spaces. A bounded linear operation $A$ from $\mathbb{H}$ into $\ell_{2}(\mathbb{H})$ can be realized as

$$
A=\left(\begin{array}{c}
A_{1} \\
A_{2} \\
\vdots
\end{array}\right)
$$

with

$$
A(x)=\left(\begin{array}{c}
A_{1}(x) \\
A_{2}(x) \\
\vdots
\end{array}\right) \quad \& \quad\left\|\sum_{i} A_{i}^{*} A_{i}\right\|^{\frac{1}{2}}=\|A\| .
$$

Thus $A^{*}$ from $\ell_{2}(\mathbb{H})$ into $\mathbb{H}$ can be realized as $\left(A_{1}^{*}, A_{2}^{*}, \ldots\right)$ with

$$
A^{*}\left(\begin{array}{c}
x_{1} \\
x_{2} \\
\vdots
\end{array}\right)=\sum_{i} A_{i}^{*}\left(x_{i}\right),
$$

convergence in norm topology.

Let $W=\left(w_{i j}\right)$ be a bounded linear operator on $\ell_{2}$, which can be identified with $W \otimes I$ on $\ell_{2}(\mathbb{H})$. In this section we will assume this identification without further warning. 
2.2 Definition. A family of operators $\left\{A_{i j}\right\}_{1 \leqslant i, j<\infty}$ in $B(\mathbb{H})$ is said to be strongly independent, if $\sum_{i, j} w_{i j} A_{i j}=0$ for some $W=\left(w_{i j}\right)$ in $B\left(\ell_{2}\right)$, the convergence in strong operator topology, implies $w_{i, j}=0$ for all $1 \leqslant i, j<\infty$. A sequence $\left\{A_{i}\right\}$ of operators is said to be strongly independent if $\sum_{i} \omega_{i} A_{i}=0$ for $\left(\omega_{i}\right) \in \ell_{2}$ implies $\omega_{i}=0$ for all $i=1,2, \ldots$.

In this notation, we have the following theorems. For simplicity, we assume Hilbert spaces to be separable.

2.3 Theorem. Every normal element in $P_{\infty}(B(\mathbb{H}))_{R}$ takes the form

$$
\Phi(x)=A^{*} \pi(x) A, \text { for all } x \text { in } B(\mathbb{H}),
$$

where $\pi$ is a representation of $B(\mathbb{H})$ on $\ell_{2}(\mathbb{H})$ by ampliation, i.e., $\pi(x)=1 \otimes x$, and

$$
A=\left(\begin{array}{c}
A_{1} \\
A_{2} \\
\vdots
\end{array}\right)
$$

$\left\{A_{i}\right\} \subseteq R^{\prime}$, the commutant of $R$, is strongly independent with $\|\Phi\|_{c b}=\left\|\sum A_{i}^{*} A_{i}\right\|$.

Proof. Since $\Phi$ is normal, it follows that $\Phi$ is the normal extension of the restriction, $\phi$, of $\Phi$ to $K(\mathbb{H})$, the $C^{*}$-algebra of all compact operators on $\mathbb{H}$. By Stinespring's representation theorem for completely positive linear maps, we have $\phi(\cdot)=u^{*} \pi(\cdot) u$, where $\pi$ is a representation of $K(\mathbb{H})$ on some Hilbert space $\mathbb{H}_{1}, u: \mathbb{H}: \rightarrow \mathbb{H}_{1}$ and $\|u\|^{2}=\|\phi\|_{c b}$. All irreducible representations of $K(\mathbb{H})$ are unitarily equivalent to the identity representation, and thus in this case $\phi$ has the form $\phi(\cdot)=A^{*} \pi(\cdot) A$, where $A \in B\left(\mathbb{H}, \ell_{2}(\mathbb{H})\right)$. Therefore, $\Phi$ has the form

$$
\Phi(x)=A^{*} \pi(x) A, \text { for all } x \text { in } B(\mathbb{H})
$$

where

$$
A=\left(\begin{array}{c}
A_{1} \\
A_{2} \\
\vdots
\end{array}\right)
$$

In $[12,3.1]$, Smith showed that any completely bounded left- $S$ right- $T$ module normal map $\Phi$ of $B(\mathbb{H})$ into itself with $S \subseteq B(\mathbb{H}), T \subseteq B(\mathbb{H}))$ is of the form $\Phi(x)=\sum_{i=1} s_{i} x t_{i}$ for all $x \in B(\mathbb{H})$ with $\left\{s_{i}\right\}$ and $\left\{t_{i}\right\}$ in the commutants $S^{\prime}$ and $T^{\prime}$ respectively such that $\left\|\sum_{i} s_{i} s_{i}^{*}\right\|\left\|\sum_{i} t_{i} t_{i}^{*}\right\|=\|\Phi\|^{2}$, and both $\left\{s_{i}\right\}$ and $\left\{t_{i}\right\}$ are strongly independent. Thus, in this case it implies that $\left\{A_{i}\right\} \subseteq R^{\prime}$ is strongly independent. We also note that $\left\{A_{i}\right\}$ may be a finite sequence consisting of $n$ elements, and in that case $\ell_{2}$ is $n$-dimensional.

Q.E.D.

A similar result was also proved in [7]. Now we may state the main result of this section.

2.4 Theorem. Suppose $\Phi$ is a normal completely positive $R$-module map on $B(\mathbb{H})$ and $\Phi$ takes the form as in Theorem 2.3. $\Phi$ is extreme in $P_{\infty}(B(\mathbb{H}), \Phi(1))_{R}$ if and only if the family $\left\{A_{i}^{*} A_{j}\right\}_{1 \leqslant i, j<\infty}$ is strongly independent.

Proof. Let $W=\left(w_{i j}\right)$ be a bounded linear operator on $\ell_{2}$ which is identified with $W \otimes I$ on $\ell_{2}(\mathbb{H})$. Then $W A$ is a bounded linear operator of $\mathbb{H}$ into $\ell_{2}(\mathbb{H})$ which can 
be realized as

$$
W A=\left(\begin{array}{c}
T_{1} \\
T_{2} \\
\vdots
\end{array}\right)
$$

with

$$
T_{i}=\sum_{j} w_{i j} A_{j} \quad \text { for } \quad i=1,2, \ldots
$$

with the convergence in strong operator topology.

The proof for " $\Leftarrow$ ". Suppose $\Phi=t \Phi_{1}+(1-t) \Phi_{2}$ for $0 \leqslant t \leqslant 1$ and $\Phi_{k} \in$ $P_{\infty}(B(\mathbb{H}), p)_{R}$ with $p=\Phi(I)$. By a theorem of Arveson (Theorem 1.4 .2 in [1]), there exist positive operators $S_{k} \in B\left(\ell_{2}(\mathbb{H})\right) \cap(\pi(B(\mathbb{H})))^{\prime}$ such that

$$
\Phi_{k}(x)=A^{*} S_{k}^{\frac{1}{2}} \pi(x) S_{k}^{\frac{1}{2}} A \quad(x \in B(\mathbb{H})), k=1,2 .
$$

Since $S_{k} \in(\pi(B(\mathbb{H})))^{\prime} \simeq B\left(\ell_{2}\right)$, we have $S_{k}=\left(s_{i j}^{k}\right)$ for $k=1,2$. From $\Phi_{k}(1)=$ $\Phi(1)$, we have $A^{*} A=A^{*} S_{k} A, k=1,2$. Thus,

$$
\begin{aligned}
\sum_{i} A_{i}^{*} A_{i} & =\sum_{i} A_{i}^{*}\left(\sum_{j} s_{i j}^{k} A_{j}\right) \\
& =\sum_{i, j} s_{i j}^{k} A_{i}^{*} A_{j} .
\end{aligned}
$$

Since $\left\{A_{i}^{*} A_{j}\right\}$ is strongly independent, we have

$$
s_{i j}^{k}=\delta_{i j}, \quad 1 \leqslant i, j<\infty, \quad k=1,2 .
$$

Thus $S^{k}=I$ and $\Phi=\Phi_{k}, k=1,2$.

The proof for " $\Rightarrow$ ". Suppose $\sum_{i, j} w_{i j} A_{j}^{*} A_{i}=0$ for some bounded linear operator $W=\left(w_{i j}\right) \in B\left(\ell_{2}\right)$. We may assume that $W^{*}=W \&\|W\| \leqslant 1$ without loss of generality. Let $I+W=V^{*} V, I-W=U^{*} U$ with $V=\left(v_{i j}\right), U=\left(u_{i j}\right)$ in $B\left(\ell_{2}\right)$. Identify $U=U \otimes I, V=V \otimes I$. Again by the same theorem of Arveson, $\Phi_{1}(x) \triangleq A^{*} V^{*} \pi(x) V A, \Phi_{2}(x) \triangleq A^{*} U^{*} \pi(x) U A(x \in B(\mathbb{H}))$ are completely positive and $\frac{1}{2}\left(\Phi_{1}(x)+\Phi_{2}(x)\right)=A^{*}\left(\frac{V^{*} V+U^{*} U}{2}\right) \pi(x) A=A^{*} \pi(x) A=\Phi(x)$.

We have

$$
U A=\left(\begin{array}{c}
U_{1} \\
U_{2} \\
\vdots
\end{array}\right), \quad V A=\left(\begin{array}{c}
V_{1} \\
V_{2} \\
\vdots
\end{array}\right)
$$

with both $U_{i}=\sum_{j} u_{i j} A_{j}$ and $V_{i}=\sum_{j} v_{i j} A_{j}$ in $R^{\prime}$ for $i=1,2$. Thus $\Phi_{k} \in$ $P_{\infty}(B(\mathbb{H}))_{R}, \frac{1}{2} \Phi_{k} \leqslant \Phi, k=1,2$, and $\Phi_{k}(I)=A^{*} V^{*} V A$ (or $\left.=A^{*} U^{*} U A\right)=$ $A^{*}(I \pm W) A=A^{*} A \pm A^{*} W A=A^{*} A=\Phi(I), \quad k=1,2$. By the extremeness assumption of $\Phi$, we have $\Phi_{1}=\Phi_{2}=\Phi$. Hence

$$
A^{*} \pi(x) A=A^{*} V^{*} V \pi(x) A=A^{*} U^{*} U \pi(x) A
$$

for all $x$ in $B(\mathbb{H})$. 
Now, it remains to be shown that $U^{*} U=V^{*} V=I$, from which it follows that $W=0$. We need just to show $U^{*} U=I$ and then $V^{*} V=I$ will follow by the same proof. Suppose that $U^{*} U \neq I$. Then there exists $\xi \in \ell_{2}$ such that $U^{*} U \xi \neq \xi$. We denote $U^{*} U \xi$ by $\eta$. For

$$
w=\left(w_{1}, w_{2}, \ldots\right) \in \ell_{2} \quad \text { and } \quad A=\left(\begin{array}{c}
A_{1} \\
A_{2} \\
\vdots
\end{array}\right),
$$

we denote

$$
\left(\begin{array}{c}
w_{1} A_{1} \\
w_{2} A_{2} \\
\vdots
\end{array}\right)
$$

by $w A$. Since $\left\{A_{i}\right\}$ is strongly independent, $\left\{A_{i}^{*}\right\}$ is strongly independent. Thus, we have

$$
\sum_{i}\left\langle\eta_{i} A_{i}^{*}\left(\theta_{1}\right), \theta_{2}\right\rangle \neq \sum_{i}\left\langle\xi_{i} A_{i}^{*}\left(\theta_{1}\right), \theta_{2}\right\rangle
$$

for some $\theta_{1}, \theta_{2} \in \mathbb{H}$. By (2.5), we have $A^{*} \pi(x) \xi A=A^{*} \pi(x) U^{*} U \xi A$ for all $x \in B(\mathbb{H})$. Thus, letting $x=\theta_{1} \odot \theta_{3}$, a rank-one projection operator in $B(\mathbb{H})$, we have

$$
\begin{aligned}
& \left\langle A^{*}\left(I \otimes\left(\theta_{1} \odot \theta_{3}\right) \eta A\right) \theta_{4}, \theta_{2}\right\rangle=\left\langle A^{*}\left(I \otimes\left(\theta_{1} \odot \theta_{3}\right) \xi A\right) \theta_{4}, \theta_{2}\right\rangle, \\
& \sum_{i}\left\langle A_{i} \theta_{4}, \theta_{3}\right\rangle\left\langle\eta_{i} A_{i}^{*} \theta_{1}, \theta_{2}\right\rangle=\sum_{i}\left\langle A_{i} \theta_{4}, \theta_{3}\right\rangle\left\langle\xi_{i} A_{i}^{*} \theta_{1}, \theta_{2}\right\rangle
\end{aligned}
$$

for $\theta_{1}, \theta_{2}, \theta_{3}, \theta_{4} \in \mathbb{H}$. Let $\theta_{3}, \theta_{4}$ vary, and we have

$$
\sum_{i} A_{i}\left\{\left\langle\eta i A_{i}^{*} \theta_{1}, \theta_{2}\right\rangle-\left\langle\xi_{i} A_{i}^{*} \theta_{1}, \theta_{2}\right\rangle\right\}=0
$$

Since $\left\{A_{i}\right\}$ is strongly independent, it follows that $\left\langle\eta_{i} A_{i}^{*} \theta_{1}, \theta_{2}\right\rangle=\left\langle\xi_{i} A_{i}^{*} \theta_{1}, \theta_{2}\right\rangle$ for all $\theta_{1}, \theta_{2} \in \mathbb{H}$, which contradicts (2.6). Therefore, $U^{*} U=I$. This completes the proof of Theorem 2.4.

Q.E.D.

3. Characterization of pure elements in $P_{\infty}(A, B)$ AND EXTREME ELEMENTS IN $P_{\infty}(A, B, p)$

For $\Phi$ in $P_{\infty}(A, B)$, we denote by $[0, \Phi]$ the set of all elements $\Psi$ in $P_{\infty}(A, B)$ with $\Phi-\Psi$ in $P_{\infty}(A, B)$. An element $\Phi$ in $P_{\infty}(A, B)$ is called pure, if $[0, \Phi]=$ $\{\lambda \Phi \mid 0 \leqslant \lambda \leqslant 1$, scalars $\}$. Here we first give the definition of a Hilbert module $\mathbb{H}$ over a $C^{*}$-algebra $B[11]$.

3.1 Definition. A pre-Hilbert $B$-module is a right $B$-module $X$ equipped with a conjugate bilinear map $\langle\cdot, \cdot\rangle: X \times X \rightarrow B$ satisfying

(i) $\langle x, x\rangle \geqslant 0$ for all $x \in X$;

(ii) $\langle x, x\rangle=0$ only if $x=0$;

(iii) $\langle x, x\rangle=\langle y, x\rangle^{*}$ for all $x, y \in X$;

(iv) $\langle x \cdot b, y\rangle=\langle x, y\rangle b$ for all $x, y \in X, b \in B$. 
For $x \in X$, put $\|x\|=\|\langle x, x\rangle\|^{\frac{1}{2}}$. A pre-Hilbert $B$-module which is complete in this norm topology is called a Hilbert $B$-module.

Let $\mathbb{H}$ be a Hilbert $B$-module, and $\mathbb{H}^{\#}$ be the Banach space of all bounded $B$ module maps of $\mathbb{H}$ into $B$. $\mathbb{H}^{\#}$ becomes a $B$-module under the definition: for all $b \in B, \lambda \in \mathbb{H}^{\#}, h \in \mathbb{H},(\lambda \cdot b)(h) \triangleq b^{*} \lambda(h)$. $\mathbb{H}$ is called self-dual, if $\mathbb{H}^{\#}=\mathbb{H}$ as a $B$-module. We denote the set of all bounded $B$-module maps of $\mathbb{H}$ into $\mathbb{H}^{\#}$ by $B\left(\mathbb{H}, \mathbb{H}^{\#}\right)$ and the set of all bounded $B$-module maps of $\mathbb{H}$ into itself by $B(\mathbb{H})$. As in [11], we denote by $L(\mathbb{H})$ the subset of $B(\mathbb{H})$ consisting of $T \in B(\mathbb{H})$ such that there exists $T^{*} \in B(\mathbb{H})$ satisfying $\langle T(x), y\rangle=\left\langle x, T^{*}(y)\right\rangle$ for all $x, y \in \mathbb{H}$. Actually $T^{*}$ is also in $L(\mathbb{H}) . \quad L(\mathbb{H})=B(\mathbb{H})$, if $\mathbb{H}$ is self-dual, and in that case $L(\mathbb{H})$ is a $W^{*}$-algebra. $A$ representation $\pi$ of a $C^{*}$-algebra $A$ on a Hilbert $B$-module $\mathbb{H}$ is a *-homomorphism of $A$ into $L(\mathbb{H})$. Given a Hilbert $B$-module $\mathbb{H}$, a representation $\pi$ of $A$ on $\mathbb{H}$ and a vector $e$ in $\mathbb{H}$, one can define a completely positive map $\Phi$ of $A$ into $B$ as $\Phi(a)=\langle\pi(a), e, e\rangle$ for all $a \in A$. In [11] the converse is proved for unital $C^{*}$ algebras $A, B$ as follows. The following theorem is Theorem 5.2 in [11].

3.2 Theorem. Let $A$ and $B$ be unital $C^{*}$-algebras and $\Phi$ be in $P_{\infty}(A, B)$. Then there is a Hilbert $B$-module $\mathbb{H}_{\Phi}$, a representation $\pi_{\Phi}$ of $A$ on $\mathbb{H}_{\Phi}$ and an element $e$ in $\mathbb{H}_{\Phi}$ such that $\Phi(a)=\left\langle\pi_{\Phi}(a) e, e\right\rangle$ for all $a \in A$, and the set $\left\{\pi_{\Phi}(a)(e \cdot b) \mid a \in\right.$ $A, b \in B\}$ spans a dense subspace $\mathbb{H}_{0}$ of $\mathbb{H}_{\Phi}$.

The Hilbert $B$-module constructed in the above theorem is not self-dual in general. In case $B$ is a $W^{*}$-algebra, $H_{\Phi}^{\#}$ is self-dual, and elements in $B\left(\mathbb{H}_{\Phi}\right)$ can be uniquely extended to elements in $B\left(\mathbb{H}_{\Phi}^{\#}\right)$ by Proposition 3.6 in [11]. This motivates us to give the following definition.

3.3 Definition. Let $\mathbb{H}$ be a Hilbert $B$-module for a $C^{*}$-algebra $B$. The algebraic tensor product $\mathbb{H} \otimes B^{* *}$ becomes a right $B^{* *}$-module if we define $(b \otimes a) \cdot a_{1}=h \otimes a a_{1}$, for all $h \in \mathbb{H}, a, a_{1} \in B^{* *}$. Then we define a $B^{* *}$ inner product on $\mathbb{H} \otimes B^{* *}$ by

$$
\left[\sum_{i} h_{i} \otimes a_{i}, \sum_{j} g_{j} \otimes b_{j}\right] \triangleq \sum_{i, j} b_{j}^{*}\left\langle h_{i}, g_{j}\right\rangle a_{i} .
$$

Set $N=\left\{z \in \mathbb{H} \otimes B^{* *} \mid[z, z]=0\right\}$. As in $[9],\left(\mathbb{H} \otimes B^{* *}\right) / N$ becomes a pre-Hilbert $B^{* *}$-module containing $\mathbb{H}$ as a $B$-submodule. Denote the self-dual Hilbert $B^{* *}$ module $\left[\left(\mathbb{H} \otimes B^{* *} / N\right)^{-}\right]^{\#}$ by $\widetilde{\mathbb{H}}$. We may consider $\mathbb{H}$ as embedded in $\widetilde{\mathbb{H}}$ without making distinction.

Every element in $B(\mathbb{H})$ can extend uniquely to an element in $B(\widetilde{\mathbb{H}})$, for a $B$ module $\mathbb{H}$. Indeed, let $T \in B(\mathbb{H})$, and one can easily see that $T$ extends uniquely to a $B^{* *}$-module map $\hat{T}$ on $\mathbb{H} \otimes B^{* *} / N$ with $\|\hat{T}\|=\|T\|$. Then by $[11,3.6] \hat{T}$ extends uniquely to a $B^{* *}$-module map $\widetilde{T}$ in $B(\widetilde{\mathbb{H}})$. by

Suppose $T$ is in $B\left(\mathbb{H}, \mathbb{H}^{\#}\right)$. We will extend $T$ to an element in $B\left(\left(\mathbb{H} \otimes B^{* *} / N\right)^{-}, \widetilde{\mathbb{H}}\right)$

$$
\left[T\left(\sum h_{i} \otimes a_{i}\right), \quad \sum g_{j} \otimes b_{j}\right]=\sum b_{j}^{*}\left[T\left(h_{i}\right), g_{j}\right] a_{i}
$$

for $a_{i}, b_{j}$ in $A^{* *}, h_{i}, g_{j}$ in $\mathbb{H}$. Then extend it again to an element $\tilde{T}$ in $B(\widetilde{\mathbb{H}})$ by Proposition 3.6 in [11]. These two unique extensions will be used later frequently. 
In fact, for $T \in B(\mathbb{H})$ (or $B\left(\mathbb{H}, \mathbb{H}^{\#}\right)$ ) if $\left.\tilde{T}\right|_{\mathbb{H}}=0$, then consider $\widetilde{T}^{*}$ in $B(\widetilde{\mathbb{H}})$. Thus

$$
\left[\tilde{T}^{*}\left(\sum_{i} h_{i}, \otimes a_{i}\right), \quad \sum_{j} g_{j} \otimes b_{j}\right]=\sum_{i, j} b_{j}^{*}\left\langle h_{i}, T g_{j}\right\rangle a_{i}=0
$$

for all $a_{i}, b_{j}$ in $A^{* *}, h_{i}, g_{j}$ in $\mathbb{H}$. Hence $\tilde{T}^{*}=0$ and $\tilde{T}=0$. Let $\Phi$ be in $P_{\infty}(A, B)$ for unital $C^{*}$-algebras $A, B$, and let $\mathbb{H}_{\Phi}, \pi_{\Phi}$ be the Hilbert $B$-module and the ${ }^{*}$ representation constructed in Theorem 3.2. For each $0 \leqslant T \leqslant I_{\mathbb{H}_{\Phi}}$ in $B\left(\mathbb{H}_{\Phi}, \mathbb{H}_{\Phi}^{\#}\right)$ we define an element $\Phi_{T}$ in $P_{\infty}(A, B)$ as follows: $\Phi_{T}(a) \triangleq\left\langle T \pi_{\Phi}(a) e, e\right\rangle$ for $a \in A$. It turns out that $\Phi_{T} \leqslant \Phi$. In [9], Lin shows that the map $T \rightarrow \Phi_{T}$ is an affine order isomorphism of $\left\{T \in B\left(\mathbb{H}_{\Phi}, \mathbb{H}_{\Phi}^{\#}\right) \mid 0 \leqslant T \leqslant I_{\mathbb{H}_{\Phi}}, T\right.$ commutes with $\left.\pi_{\Phi}(A)\right\}$ on $[0, \Phi]$.

In this section we establish a different characterization of $[0, \Phi]$. Let $\Phi, \Psi$ be in $P_{\infty}(A, B)$ and $\Psi \leqslant \Phi$. By Theorem 3.2, $\Phi(a)=\left\langle\pi_{\Phi}(a) e \cdot e\right\rangle$ for all $a \in A$ where $\pi_{\Phi}$ is a $*$-representation in a Hilbert $B$-module $\mathbb{H}_{\Phi}$ with $e \in \mathbb{H}_{\Phi}$, and $\Psi(a)=$ $\left\langle\pi_{\Psi}(a) f, f\right\rangle$ for all $a \in A$ where $\pi_{\Psi}$ is a $*$-representation on a Hilbert $B$-module $\mathbb{H}_{\Phi}$ with $f \in \mathbb{H}_{\Psi}$. Now we define a bounded module map $W$ from $\mathbb{H}_{\Phi}$ into $\mathbb{H}_{\Psi}$ by $W\left(\pi_{\Phi}(a)(e \cdot b)\right)=\pi_{\Psi}(a)(f \cdot b)$ for all $x \in A, b \in B$ on a dense subspace $X_{\Phi}$ of $\mathbb{H}_{\Phi}$ into a dense subspace $X_{\Psi}$ of $\mathbb{H}_{\Psi}$, where $X_{\Phi}=\left\{\pi_{\Phi}(a)(e \cdot b) \mid a \in A, b \in B\right\}$, $X_{\Psi}=\left\{\pi_{\Psi}(a)(f \cdot b) \mid a \in A, b \in B\right\}$. We note that $\langle W(x), W(x)\rangle \leqslant\langle x, x\rangle$ for all $x$ in $X_{\Phi}$, because $\Psi \leqslant \Phi$. We extend $W$ to $\mathbb{H}_{\Phi}$, the extension still denoted by $W$, and note that $W$ is a module map with $\|W\| \leqslant 1$. In Definition 3.3 we can easily extend $W$ to a bounded $B^{* *}$-module (denoted by $W$ again) from $H_{\Phi} \otimes B^{* *}$ into $H_{\Psi} \otimes B^{* *}$. $W$ maps $N_{\Phi}$ into $N_{\Psi}$ because $\Psi \leqslant \Phi$. Hence we can further extend $W$ to a bounded module map (denoted by $W$ again) from $\left(\mathbb{H}_{\Phi} \otimes B^{* *} / N_{\Phi}\right)^{-}$into $\left(\mathbb{H}_{\Psi} \otimes B^{* *} / N_{\Psi}\right)^{-}$. By Proposition 3.6 [11], $W$ eventually extends to a module map $\widetilde{W}$ from $\widetilde{\mathbb{H}}_{\Phi}$ into $\widetilde{\mathbb{H}}_{\Psi}$. Thus we have the following

3.4 Theorem. Let $\Psi, \Phi$ be in $P_{\infty}(A, B)$ with $\Psi \leqslant \Phi$. Then there is a bounded $B$-module map $W$ of $\mathbb{H}_{\Phi}$ into $\mathbb{H}_{\Psi}$ such that $W \pi_{\Phi}(a)=\pi_{\Psi}(a) W$ for all $a \in A$. Furthermore, $W$ can extend uniquely to a bounded $B^{* *}$-module map $\widetilde{W}$ from $\widetilde{\mathbb{H}}_{\Phi}$ into $\widetilde{\mathbb{H}}_{\Psi}$ with $\|\widetilde{W}\|=\|W\|$.

Proof. We only need to show $W \pi_{\Phi}(a)=\pi_{\Psi}(a) W$ for all $a$ in $A$ and $\widetilde{W}$ being the unique extension of $W$ with $\|\widetilde{W}\|=\|W\|$. They are results of straightforward calculations.

Q.E.D

Let $T=\widetilde{W}^{*} \widetilde{W}$. We may extend $\pi_{\Phi}(A)$ to $\tilde{\pi}_{\Phi}(A)$ on $\widetilde{\mathbb{H}}_{\Phi}$.

3.5 Lemma. $T$ commutes with $\tilde{\pi}_{\Phi}(a)$ for all $a$ in $A$ and $\Psi(a)=\left\langle T \tilde{\pi}_{\Phi}(a) e, e\right\rangle$ for all $a \in A$.

Proof. From $W \pi_{\Phi}(a)=\pi_{\Psi}(a) W$ we can easily see $\widetilde{W} \tilde{\pi}_{\Phi}(a)=\tilde{\pi}_{\Psi}(a) \widetilde{W}$ for all $a \in A$. Thus,

$$
T \tilde{\pi}_{\Phi}(a)=\widetilde{W}^{*} \widetilde{W} \tilde{\pi}_{\Phi}(a)=\widetilde{W}^{*} \tilde{\pi}_{\Psi}(a) \widetilde{W}=\tilde{\pi}_{\Phi}(a) \widetilde{W} * \widetilde{W}=\tilde{\pi}_{\Phi}(a) T .
$$

Next we consider

$$
\left\langle T \tilde{\pi}_{\Phi}(a) e, e\right\rangle=\left\langle\widetilde{W^{*}} \widetilde{W} \tilde{\pi}_{\Phi}(a) e, e\right\rangle=\left\langle\pi_{\Psi}(a) f, \widetilde{W} e\right\rangle=\left\langle\pi_{\Psi}(a) f, f\right\rangle=\Psi(a)
$$

for all $a$ in $A$. 
Denote by $\Phi_{T}$ the map $a \mapsto\left\langle T \tilde{\pi}_{\Phi}(a) e, e\right\rangle$ for $a$ in $A$.

3.6 Theorem. There is a bijection $T \mapsto \Phi_{T}$ from the set $=\left\{T \in B\left(\widetilde{\mathbb{H}}_{\Phi}\right) \mid 0 \leqslant T \leqslant\right.$ $I, T \tilde{\pi}_{\Phi}(a)=\tilde{\pi}_{\Phi}(a) T,\left\langle T \tilde{\pi}_{\Phi}(a) e, e\right\rangle \in B$ for all a in $\left.A\right\}$ onto $[0, \Phi]$.

Proof. From the previous lemma, it is clear that for any $\Psi \in[0, \Phi]$, there is a $T \in B\left(\tilde{H}_{\Phi}\right)$ with $0 \leqslant T \leqslant 1$ such that $T \tilde{\pi}_{\Phi}(a)=\tilde{\pi}_{\Phi}(a) T,\left\langle T \tilde{\pi}_{\Phi}(a) e, e\right\rangle \in B$ for all $a$ in $A$, and $\Phi_{T}=\Psi$. It is also obvious that the map $T \rightarrow \Phi_{T}$ is order-preserving. Now we only need to show the map is one-to-one. For such $T$ and $a_{1}, \ldots, a_{n} \in A$, $b_{1}, \ldots, b_{n} \in B$, we have

$$
\begin{aligned}
\sum_{i j} b_{i}^{*} \Phi_{T}\left(a_{i}^{*} a_{j}\right) b_{j}=\sum_{i j} b_{i}^{*}\left\langle T \tilde{\pi}_{\Phi}\left(a_{i}^{*}\right) \tilde{\pi}_{\Phi}\left(a_{j}\right) e, e\right\rangle b_{j} \\
=\left\langle T^{\frac{1}{2}}\left(\sum_{j} \pi_{\Phi}\left(a_{j}\right)\left(e \cdot b_{j}\right)\right), T^{\frac{1}{2}}\left(\sum_{i} \pi_{\Phi}\left(a_{i}\right)\left(e \cdot b_{i}\right)\right)\right\rangle \geqslant 0 .
\end{aligned}
$$

Hence, $\Phi_{T}$ is in $P_{\infty}(A, B)$. Next, one can see that $T=0$, if $\Phi_{T}=0$. For

$$
\left\langle T \pi_{\Phi}\left(a_{1}\right)\left(e \cdot b_{1}\right), \pi\left(a_{2}\right)\left(e \cdot b_{2}\right)\right\rangle=0 \quad \text { for all } a_{1}, a_{2} \in A, b_{1}, b_{2} \in B,
$$

if $\Phi_{T}=0$. Then $\left.T\right|_{\mathbb{H}_{\Phi}}=0$. Since $\left\langle T \tilde{\pi}_{\Phi}(a)\left(a \cdot b_{1}\right), e \cdot b_{2}\right\rangle \in B$ and $T$ is the unique extension of $\left.T\right|_{\mathbb{H}_{\Phi}}$, it follows that $T=0$.

Q.E.D.

3.7 Corollary. Let $A, B$ be unital $C^{*}$-algebras, and $\Phi$ be in $P_{\infty}(A, B)$. Then $\Phi$ is pure if and only if the set $\left\{T \in B\left(\tilde{H}_{\Phi}\right) \mid 0 \leqslant T \leqslant I, T \tilde{\pi}_{\Phi}(a)=\tilde{\pi}_{\Phi}(a) T\right.$, $\left\langle T \tilde{\pi}_{\Phi}(a) e, e\right\rangle \in B$ for all $a$ in $\left.A\right\}$ consists of scalar multiplications of $I$ only.

Next, we characterize the set of extreme elements in $P_{\infty}(A, B, p)$. We denote by $C_{0}(\Phi)$ the positive cone generated by the set $\left\{T \in B\left(\widetilde{\mathbb{H}}_{\Phi}\right) \mid 0 \leqslant T \leqslant I, T \tilde{\pi}_{\Phi}(a)=\right.$ $\tilde{\pi}_{\Phi}(a) T,\left\langle T \tilde{\pi}_{\Phi}(a) e, e\right\rangle \in B$ for all $\left.a \in A\right\}$.

3.8 Theorem. $\Phi$ is extreme in $P_{\infty}(A, B, p)$ if and only if the map $T \mapsto\langle T e, e\rangle$ is one-to-one on $C_{0}(\Phi)$.

Proof. Suppose that $T \mapsto\langle T e, e\rangle$ is one-to-one on $C_{0}(\Phi)$. Let $\Phi=t, \Psi_{1}+(1-t) \Psi_{2}$ with $0 \leqslant t \leqslant 1, \Psi_{i} \in P_{\infty}(A, B, p)$. By Theorem 3.6 there exists $T_{i} \in B\left(\widetilde{\mathbb{H}}_{\Phi}\right)$ with $0 \leqslant T_{i} \leqslant I, T_{i} \tilde{\pi}_{\Phi}(a)=\tilde{\pi}_{\Phi}(a) T_{i},\left\langle T_{i} \tilde{\pi}_{\Phi}(a) e, e\right\rangle \in B$ for all $a \in A, i=1,2$, such that $t \Psi_{1}(a)=\left\langle T_{1} \tilde{\pi}_{\Phi}(a) e, e\right\rangle,(1-t) \Psi_{2}(a)=\left\langle T_{2} \tilde{\pi}_{\Phi}(a) e, e\right\rangle$ for all $a \in A$. Thus $t\langle e, e\rangle=t p=t \Psi_{1}(I)=\left\langle T_{1} e, e\right\rangle$. Hence $T_{1}=t I$ and $\Phi=\Psi_{1}=\Psi_{2}$.

Conversely, suppose $\Phi$ is extreme and $\langle T e, e\rangle=0$ for some $T$ in $C_{0}(\Phi)$. Choose positive scalars $s, t$ such that $\frac{1}{4} I \leqslant s T+t I \leqslant \frac{3}{4} I$. Denote $s T+t I=U$. Thus, $\langle U e, e\rangle$ $=\langle(s T+t I) e, e\rangle=t p$, with $\frac{1}{4} p \leqslant t p \leqslant \frac{3}{4} p$. Thus $0<t<1$. Define $\Psi_{1}(a)=$ $\left\langle U \tilde{\pi}_{\Phi}(a) e, e\right\rangle, \Psi_{2}(a)=\left\langle(1-U) \tilde{\pi}_{\Phi}(a) e, e\right\rangle$ for all $a \in A$. Then $\Psi_{1}+\Psi_{1}=\Phi$. Since $t^{-1} \Psi_{1}$ and $(1-t)^{-1} \Psi_{2}$ are in $P_{\infty}(A, B, p)$, it follows that $t^{-1} \Psi_{1}=(1-t)^{-1} \Psi_{2}=\Phi$, and $\Psi_{1}(a)=t \Phi(a)$, i.e. $\left\langle U \tilde{\pi}_{\Phi}(a) e, e\right\rangle=\left\langle t \tilde{\pi}_{\Phi}(a) e, e\right\rangle$ for all $a \in A$. By uniqueness of extension of elements in $B\left(\mathbb{H}_{\Phi}\right)$ to elements in $B\left(\widetilde{\mathbb{H}}_{\Phi}\right)$ we have $U=t I$. Thus $T=0$.

Q.E.D.

3.9 Concluding remarks. In Definition 3.3, there is really just a procedure for constructing $\tilde{H}$ in which $B^{* *}$ can be replaced by a $\sigma$-closure $\pi(\bar{B})$ of $\pi(B)$ under a faithful representation $\pi$ of $B$. Then Theorems 3.4, 3.6 and 3.8, Corollary 3.7, and Lemma 3.5 will still hold with this new $\overline{\mathbb{H}}$. This then suggests a new way of treating the Stinespring like representation induced by an element in $P_{\infty}(A, B)$. 


\section{REFERENCES}

1. W. Arveson, Subalgebras of $C^{*}$-algebras, Acta Math. 123 (1969), 141-224. MR 40:6274

2. M.D. Choi, Completely positive linear maps on $C^{*}$-algebras, Linear Algebra Appl. 12 (1975), 95-100. MR 52:270

3. M.D. Choi and T.Y. Lam, External positive semidefinite forms, Math. Ann. 231 (1977), 1-18. MR 58:16512

4. C. Delaroche, On completely positive maps defined by an irreducible correspondence, Canad. Math. Bull. 33 (1990), 434-441.

5. V. Haagerup, Decompositions of completely bounded maps on operator algebras, unpublished, preprint, 1980.

6. R. Kadison and J. Ringrose, Elements of the theory of operator algebras, Vol. 1, Academic Press, New York, 1983. MR 85j:46099

7. K. Kraus, Operations and effects in the Hilbert space formulation of quantum theory, Lecture Notes in Phys., vol. 29, Springer-Verlag, New York, 1974. MR 58:14672

8. S.H. Kye, A class of atomic positive maps in 3-dimensional matrix algebra, Elementary Operators and Applications, (Blauberen 1991), World Sci. Publishing, River Edge, NJ, 1992 pp. 205-209. MR 94a:15050

9. H. Lin, Bounded module maps and pure completely positive maps, J. Operator Theory 26 (1991), 73-92. MR 94f:46071

10. H. Osaka, A class of external positive in $3 \times 3$ matrix algebras, Publ. Res. Inst. Math. Sci. 28 (1992), 747-756. MR 94a:46076

11. W. Paschke, Inner product modules over $B^{*}$-algebras, Trans. Amer. Math. Soc. 182 (1973), 443-468. MR 50:8087

12. R. Smith, Completely bounded module maps and Haagerup tensor product, J. Funct. Anal. 102 (1991), 156-175. MR 93a:46115

13. E. Størmer, Positive linear maps of operator algebras, Acta Math. 110 (1963), 233-278. MR 27:6145

14. T. Takasaki and J. Tomiyama, On the geometry of positive maps in matrix algebras, Math. Z. 184 (1983), 101-108. MR 85b:46063

15. S.K. Tsui, Constructions of extreme n-positive linear maps, Contemp. Math., vol. 120, Amer. Math. Soc., Providence, RI, 1991, pp. 175-181. MR 92m:46092

16. __ Extreme n-positive linear maps, Proc. Edinburgh Math. Soc. 36 (1992), 123-131. MR 94b:46088

Department of Mathematical Sciences, Oakland University, Rochester, Michigan 48309-4401

E-mail address: tsui@vela.acs.oakland.edu 